\title{
A NEW SYSTEM OF SHORT WAVE AMPLIFICATION*
}

\author{
$\mathrm{BY}$ \\ Edwin H. Armstrong \\ (Columbia University, New York)
}

The problem of receiving weak signals of short wave length in a practical manner has become of great importance in recent years. This is especially true in connection with direction finding work where the receiver must respond to a very small fraction of the energy which can be picked up by a loop antenna.

The problem may be summed up in the following words:to construct a receiver for undamped, modulated continuous, and damped oscillations which is substantially equally sensitive over a range of wave lengths from 50 to 600 meters, which is capable of rapid adjustment from one wave to another, and which does not distort or lose any characteristic note or tone inherent in the transmitter.

It is, of course, obvious that some form of amplification must be used, but a study of the various known methods soon convinces one that a satisfactory solution cannot be obtained by any direct method. In the interests of completeness, we will consider the three well-known direct means which might possibly be employed, and examine the limitations. which apply to each. These three methods are:-

(1) Amplification of the audio frequency current after rectification;

(2) Amplification of the radio frequency current before rectification; and

(3) Application of the heterodyne principle to increase the efficiency of rectification.

Consider first the method of rectifying the radio frequency current and amplifying the resulting audio frequency current. Two limitations at once present themselves, one inherent in audio frequency amplifiers, and the other inherent in all known rectifiers. The limitation in the amplifier is the residual noise

* Presented before The Institute of Radio Engineers, New York, December 3, 1919. Received by the Editor, January 12, 1920. 
which makes it impractical to use effectively more than two stages of amplification. The second limitation lies in the characteristic of the detector or rectifier. All rectifiers have a characteristic such that the rectified or audio frequency current is roughly proportional to the square of the impressed radio frequency emf. Hence the efficiency of rectification becomes increasingly poorer the weaker the signal until a point is reached below which the detector practically ceases to respond.

The second method of attack on the problem is the amplification of the received radio frequency currents before rectification to a point where they can be efficiently dealt with by the detector. This method is ideal on long waves, and various methods of inductance, resistance, and capacity couplings have been successfully used, but when the attempt is made to use the same methods of coupling on wave lengths below 600 meters, it results in complete failure. This is because the low capacity reactance existing between the various elements of the tubes causes them, in effect, to act as a short circuit around the coupling means and thereby prevents the establishment of a difference of potential in the external plate circuit. It is, of course, possible to eliminate the short-circuiting by tuning with a parallel inductance but this introduces a complication of adjustment which is highly objectionable and the tuning of all circuits also leads to difficulty with undesirable internal oscillations.

The third method which might be used is the heterodyne method to increase the efficiency of rectification. Great increase in signal strength is possible by means of this method, particularly where the signal is very weak, but there are certain reasons why it cannot be effectively used in practice at the present time. The chief reason in receiving continuous waves of short wave length is the instability of the beat tone which makes operations below 600 meters unsatisfactory. This disadvantage does not apply to the reception of spark signals but here the loss of the clear tone and its individuality offsets much of the gain due to increased signal strength. In the case of telephony the distortion which always results likewise offsets the gain in strength. It is, of course, undeniable that there are many special cases where the use of the heterodyne on short wave lengths is of the greatest advantage but the foregoing remarks apply to the broad field of commercial working where the practical aspects of the case greatly reduce the value of the amplification obtained by this method.

In spite of the great difficulties involved in a direct solu- 
tion, great success was obtained by Round in England and Latour in France in the production of radio frequency amplifiers to cover effectively a range from 300 to 800 meters. This result was accomplished only by the most painstaking and careful experiment and it represents some of the very finest radio work carried out during the war. Round secured his solution by constructing tubes having an extremely small capacity without increase in internal resistance above normal values and coupling the tubes by means of transformers wound with very fine wire to keep down the capacity and very high resistance to prevent oscillation at the resonant frequency of the system. The effect of the high ratio of inductance to capacity and the high resistance of the winding is to flatten the resonance curve of the system and widen the range of response. Latour solved the problem by the use of iron core transformers wound with very fine wire, the iron serving the double purpose of increasing the ratio of inductance to capacity and introducing resistance into the system. Both these factors widen the range of response.

It is the purpose of this paper to describe a method of reception evolved at the Division of Research and Inspection of the Signal Corps, American Expeditionary Force, which solves the problem by means of an expedient. This expedient consists in reducing the frequency of the incoming signal to some predetermined super-audible frequency which can be readily amplified, passing this current thru an amplifier, and then detecting or rectifying the amplified current. The transform tion of the original radio frequency to the pre-determined value is best accomplished by means of the heterodyne and rectification, and the fundamental phenomena involved will be understood by reference to the diagram of Figure 1. Here $L C$ represents the

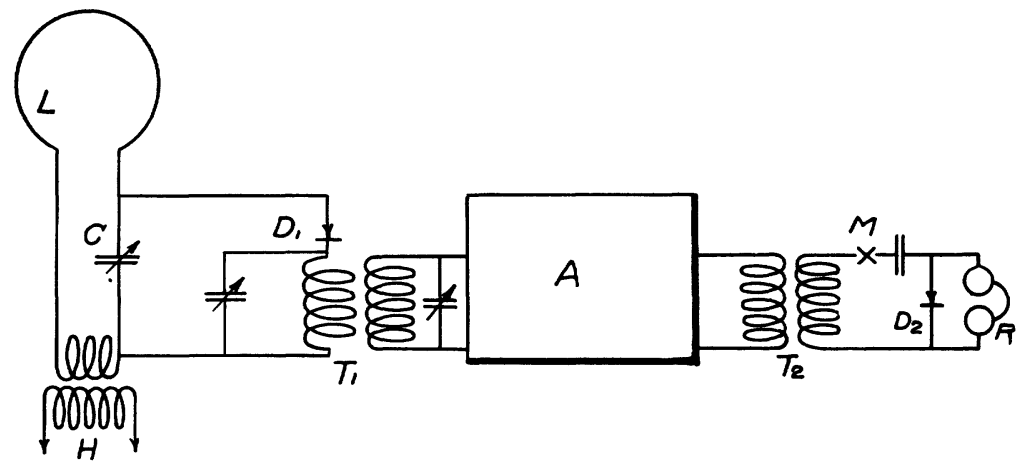

Figure 1 
usual tuned receiving circuit, loop or otherwise, $H$ a separate heterodyne, and $D_{1}$ a rectifier. $A$ is a radio frequency amplifier designed to operate on some pre-determined frequency. This frequency may be any convenient frequency which is substantially above audibility. The amplifier is connected on its input side to the rectifier $D_{1}$, and on its output side to a second rectifier $D_{2}$ and a telephone or other receiver.

Suppose now that the frequency to be received is $3,000,000$ cycles per second corresponding to a wave length of 100 meters and, for the sake of simplicity, that the incoming waves are undamped. Also, assume that the amplifier $A$ has been designed for maximum efficiency at 100,000 cycles per second. The circuit $L C$ is tuned to $3,000,000$ cycles, and the heterodyne $H$ is adjusted to either $3,100,000$ or $2,900,000$ cycles either of which will produce a beat frequency of 100,000 cycles per second. The combined currents of $3,000,000$ and $3,100,000$ (or 2,900,000) cycles are then rectified by the rectifier $D_{1}$ to produce in the primary of the transformer $T_{1}$ a direct current with a superimposed 100,000-cycle component. This 100,000-cycle current is then amplified to any desired degree by the amplifier $A$ and detected or rectified by $D_{2}$. In order to get an audible tone where telephone reception is used some form of modulation or interruption must, of course, be employed in connection with this second rectification as the current in the output circuit of the amplifier is of a frequency above audibility. While this frequency is only 100,000 cycles and while it is therefore well within the range of practical heterodyning, its steadiness depends on the beats between 3,000,000 and 3,100,000 eycles per second and hence in any attempt to heterodyne it to audibility the same difficulties due to fluctuation would be encountered as in heterodyning the original radio frequency to audibility. However, the inability to use the heterodyne on the second rectification is not of great importance because the amplitude of the signal to be rectified is large and hence the difference (as far as signal strength in the telephone is concerned) between heterodyne and modulated reception is not great.

It is important to note here that the value of the heterodyne current in the first rectifier should always be kept at the optimum value in order to ensure the carrying out of the first rectification at the point of maximum efficiency. This adjustment, however, is not a critical one, and, once made, it is seldom necessary to change it. The amplifier $A$ may be made selective and highly regenerative if so desired, and some very great increases in the 
selectivity of the system as a whole can be secured. Figure 2 illustrates the principle involved. This arrangement is substantially the same as Figure 1 except that the primary and secondary coils of the transformer $T_{1}$ are tuned by means of condensers as shown and the coupling between them is reduced to the proper value to insure sharp tuning. This system of

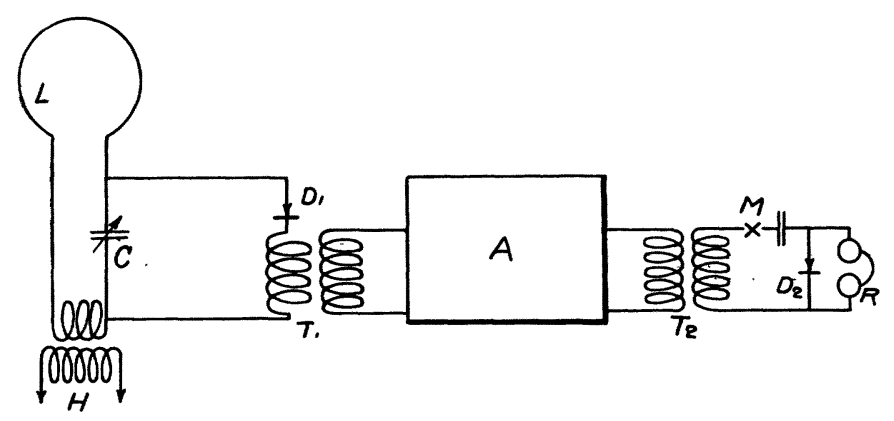

Figure 2

connection has all the advantages of tuning to the differential frequency in the manner well known in the art and an additional one due to the fact that since it is above audibility the musical character of atmospheric disturbances so troublesome in audio frequency tuning, does not appear.

So far, the reception of undamped waves only has been considered, but this method of amplification is applicable also to the reception of damped wave telegraphy and to telephony with practically equal efficiency and without distortion of any characteristics of tone. It is somewhat difficult to understand this, particularly in the case of the reception of spark signals as in all previous experience the heterodyning of a spark signal has resulted in the loss of the note, whereas in the present case the individuality between stations is more marked even than on a crystal rectifier.

This is the most interesting point in the operation of the system and the reason will be understood from the following analysis:

In heterodyning, the efficiency of rectification of the signaling current depends on its phase relation with the local current. If the two currents are either in phase or $180^{\circ}$ out of phase the efficiency of rectification is a maximum; if $90^{\circ}$ out of phase a min- 
imum. In ordinary heterodyning, the initial phase difference depends on the time of sparking at the transmitter and hence this initial phase difference will be different for each wave train. As the frequency of the two currents are substantially the same, and as the duration of a wave train is short compared to the time necessary to produce a complete beat at an audible frequency, this initial phase difference is maintained thruout the wave train. Hence, the different wave trains are rectified with varying efficiency, the telephone current becomes irregular, and a rough or hissing tone results.

In the present method of heterodyning, the beat frequency is high so that several beats per wave train are produced. As a consequence, the phase angle between the signaling and local currents varies thru several cycles and the initial phase difference becomes a matter of minor importance. The number of beats which actually occur in practice depends on the beat frequency, the damping of the incoming wave, and the damping of the receiving circuit. As the damping of the receiving circuit is almost invariably much less than the damping of the incoming wave, it is the determining factor. In any practical case, however, where the beat frequency is kept.above 20,000 cycles per second there is a sufficient number of beats to minimize the initial phase differences and maintain the characteristic tone.

The phenomena which occur in the reception of modulated continuous wave telegraphy and telephony are substantially a combination of those explained in the cases of undamped and damped wave reception. The adjustments are made in the same manner as for damped waves and the only precaution necessary in the reception of telephony is to damp the amplifier circuits somewhat to prevent distortion of the speech by excessive resonance.

The general arrangement found most suitable for practical working is shown in Figure 3. Both rectifications are carried out by three-element vacuum tubes. The amplifier here shown is resistance coupled, although any form of coupling may be used. The tuned circuits $L C$ and $L_{2} C_{2}$ are preferably adjusted to some frequency between 50,000 and 100,000 cycles. The circuit $L C$ may be made regenerative, if so desired, by any form of reactive coupling, but the practicability of this depends largely on the amount of time which is available for making adjustments.

In the diagram of Figure 3, only two stages of radio frequency amplification are shown, but at least four and preferably six should be used to get the maximum advantage of this method. 
This is because the transformation of frequency is accomplished only by a certain loss so that something between one and two stages of amplinication is required before this is overcome and it is possible to realize a gain. In this figure a separate heterodyne is shown, and it will generally be necessary to use it on account of the mistuning which is involved in the use of the self heterodyne This mistuning is considerable on 600 meters but on the shorter waves it is possible to use the self heterodyne method with equal efficiency as far as signal strength is concerned and a great gain in simplicity, as adjustments have been reduced to the minimum of a single one.

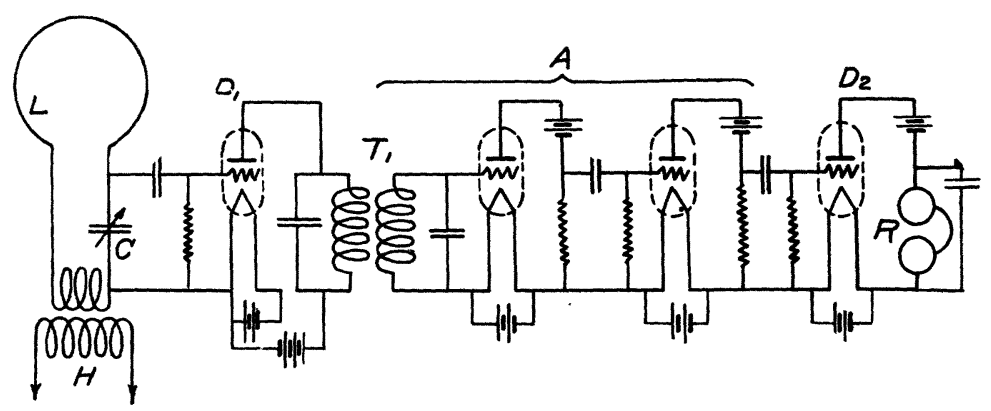

Figure 3

It may be observed here that this method is not limited to one transformation of frequency with one subsequent amplification. If the frequency to be received is $5,000,000$ cycles this may be stepped down to 500,000 cycles, amplified, stepped down again to 50,000 cycles, re-amplified and detected. The great advantage of this method of amplification is that the tendency to oscillate due to the reaction between the output of the amplifier and the input is eliminated as the frequencies are widely different. The only reaction which can take place is in each individual amplifier. Hence, the process of extreme amplification is best carried out in stages of several frequencies, the amplification on each frequency being carried as far as possible without loss of stability. As soon as the limit of stable operation is approached, no further amplification should be attempted until the frequency has been changed.

The foregoing descriptions and explanations do not pretend to any save a most superficial treatment of the phenomena present in this method of reception. Lack of time has prevented a care- 
ful study and quantitative date only of the roughest sort has been obtained. Sufficient work has been done, however, to demonstrate the value of the method particularly in the case of modulated continuous wave telegraphy and telephony. In this field neither the amplification nor the selectivity can be equalled by any direct method.

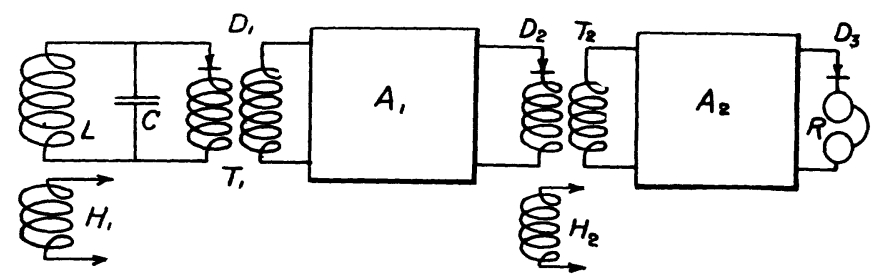

Figure 4

The practical results which have been obtained may perhaps be of interest. With a ten-turn, three-foot (1 meter) loop antenna and an amplifier consisting of six stages, resistence coupled, making a total of eight tubes, the night signals of ships working with the Florida and Gulf stations are loudly received. The night signals of amateur stations in the Middle West are regularly received as are also the signals of stations in the Gulf States. The general arrangement of the apparatus used is shown in Figures 5 and 6 which illustrate the scheme of connections of the frequency transformer and amplifier respectively. Four stages of amplification only are shown but six were actually used.

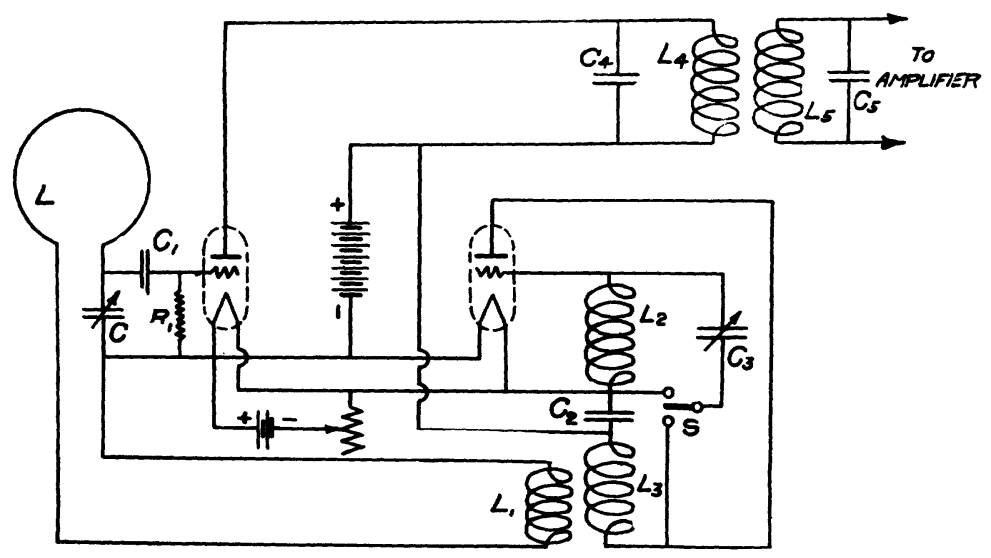

Figure 5 
It is beyond question much more efficient to use some form of inductive coupling since the amplifier is intended to operate on only one frequency and the use of a resistance coupled amplifier is not recommended where one of the former type is available.

The new practice of this method involves the use of many known inventions, but in connection with the production of a superaudible frequency by heterodyning I wish to make due acknowledgment to the work of Meissner, Round, and Levy, which is now of record. The application of the principle to the reception of short waves is, I believe, new and it is for this reason that this paper is presented.

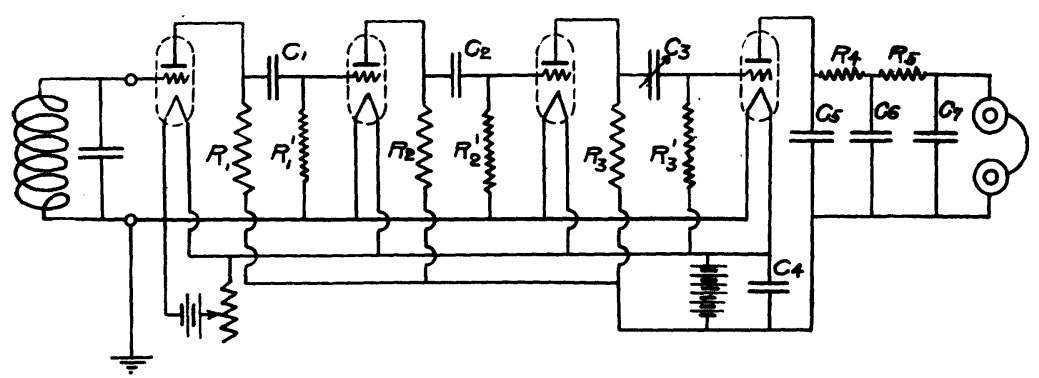

Figure 6

While the fundamental idea of this method of reception is relatively simple, the production of the present form of the apparatus was a task of the greatest difficulty for reasons known only too well to those familiar with multi-stage amplifiers; and to Lieutenant W. A. MacDonald, Master Signal Electricians J. Pressby and H. W. Lewis, and Sergeant H. Houck, all of the Division of Research and Inspection Signal Corps A. E. F., I wish to give full credit for its accomplishment.

Hartley Research Laboratory, Columbia University, New York City.

SUMMARY: The various possible known methods of amplifying incoming signals of very short wave length (below 600 meters) are described and their limitations considered.

The new method then described consists (for continuous wave reception) of the following steps:-

1. Heterodyning, with the production of a beat frequency which is itself a radio frequency (for example, 100,000 cycles per second).

2. Rectification of the beat current.

3. Amplification at the beat radio frequency, preferably by a tuned amplifier.

4. Audio frequency modulation of the amplified current.

5. Rectification of the modulated current.

For reception of damped wave or radiophone signals, step 4 is omitted. It is shown that in this case the quality (characteristic tone) of the incoming signals is preserved. 